\title{
Age dependency of peripheral and central systolic blood pressures: cross-sectional and longitudinal observations in a Chinese population
}

\begin{abstract}
Yan $\mathrm{Li}^{1}$, Jan A Staessen ${ }^{2,3}$, Chang-Sheng Sheng ${ }^{1}$, Qi-Fang Huang ${ }^{1}$, Michael O’Rourke ${ }^{4}$ and Ji-Guang Wang ${ }^{1}$
Few studies have described the age-related changes in both peripheral and central systolic blood pressures (SBPs) in populations. We addressed this issue in 1066 women and 978 men, all untreated (mean age, 45.1 years; $27.2 \%$ hypertensive) and randomly selected from a Chinese population, of whom 369 and 330 underwent a repeat examination after 3.6 years (median). In cross-sectional analyses, central SBP increased more with age than peripheral SBP in women below age 50 (1.21 vs. $1.01 \mathrm{~mm} \mathrm{Hg}$ per year; $P<0.001)$ and in men below age $60(0.73$ vs. $0.48 \mathrm{~mm} \mathrm{Hg}$ per year; $P<0.001)$, whereas in older women (0.64 vs. $0.58 \mathrm{~mm} \mathrm{Hg}$ per year; $P=0.27)$ and older men $(0.45$ vs. $0.44 \mathrm{~mm} \mathrm{Hg}$ per year; $P=0.79)$, the slopes of central and peripheral SBPs on age were similar. Compared with men, women had steeper $(P<0.001)$ age-related increases in peripheral and central SBPs. Systolic augmentation pressure increased with age, but this increase was substantially smaller $(P<0.0001)$ for peripheral than central augmentation (women, 0.086 vs. $0.45 \mathrm{~mm} \mathrm{Hg}$ per year; men, $0.083 \mathrm{vs}$. $0.39 \mathrm{~mm} \mathrm{Hg}$ per year). In multivariable-adjusted regression, age contributed $\geqslant 89.7 \%$ of the explained variance in peripheral and central SBPs. In longitudinal analyses, the annual percentage increases from baseline to follow-up in peripheral and central SBP were similar $(P \geqslant 0.76)$ in both women $(2.14 \%$ vs. $2.16 \%$ per year) and men $(1.33 \%$ vs. $1.34 \%$ per year; $P$-values for sex difference $\leqslant 0.044)$. In conclusion, in younger subjects assessed cross-sectionally, the age-related increase was larger for central than peripheral SBP, whereas the corresponding cross-sectional estimates in older subjects and the longitudinal estimates in all subjects showed similar age-related increases in central and peripheral SBP.
\end{abstract}

Hypertension Research (2012) 35, 115-122; doi:10.1038/hr.2011.160; published online 15 September 2011

Keywords: aging; central blood pressure; epidemiology; peripheral blood pressure; risk factors

\section{INTRODUCTION}

The arterial pressure wave consists of a forward component generated by the heart and reflected waves returning to the heart from peripheral sites. ${ }^{1}$ As the arteries become stiffer with advancing age, the reflected waves return faster, reach the proximal aorta during systole, and cause augmentation of late systolic blood pressure (SBP), whereas diastolic pressure decreases. Arterial stiffening and wave reflections explain why in young subjects, SBP in the brachial artery is higher than in the ascending aorta, whereas in older subjects, both values tend to become similar. Systolic augmentation is now a generally accepted physiological concept. However, the initial evidence supporting the principle of the age dependency of amplification of the central BP came from animal experiments ${ }^{2}$ and invasive studies in selected subjects. ${ }^{2-4}$ Among the published population studies on the age dependency of arterial stiffness, ${ }^{5-10}$ most focused on pulse wave velocity, ${ }^{5-8}$ the ratio of pulse pressure in peripheral vs. central arteries, ${ }^{10}$ or the augmentation index ${ }^{7,9}$ in selected healthy subjects ${ }^{7-10}$ and patients at increased cardiovascular risk. ${ }^{11}$

We previously reported age-specific reference values for the peripheral and central pulse pressures and augmentation indexes in a healthy Chinese reference population. ${ }^{9}$ In the present analyses, we focused on the changes with age in peripheral and central SBPs, systolic amplification and the difference between the augmented and non-augmented SBP. We assessed these age-related changes cross-sectionally in Chinese, as well as longitudinally in a subsample. We upfront excluded patients on antihypertensive drug treatment from analysis.

\section{METHODS}

Study population

From 2003 through 2009, we recruited participants from 18 villages in JingNing County, a rural area approximately $500 \mathrm{~km}$ south of Shanghai. The Ethics Committee of Ruijin Hospital and Shanghai Jiaotong University School of

\footnotetext{
${ }^{1}$ Center for Epidemiologic Studies and Clinical Trials and Center for Vascular Evaluations, Shanghai Key Laboratory of Vascular Biology, Shanghai Institute of Hypertension, Ruijin Hospital, Shanghai Jiaotong University School of Medicine, Shanghai, China; ${ }^{2}$ Studies Coordinating Centre, Division of Hypertension and Cardiovascular Rehabilitation, Department of Cardiovascular Diseases, University of Leuven, Leuven, Belgium; ${ }^{3}$ Department of Epidemiology, Maastricht University, Maastricht, The Netherlands and ${ }^{4}$ Saint Vincent's Clinic, University of New South Wales, Sydney, Australia

Correspondence: Dr Y Li, Center for Epidemiologic Studies and Clinical Trials and Center for Vascular Evaluations, Shanghai Key Laboratory of Vascular Biology, Shanghai Institute of Hypertension, Ruijin Hospital, Shanghai Jiaotong University School of Medicine, 197 Ruijin 2nd Road, Shanghai 200025, China.

E-mail: liyanshcn@yahoo.com
}

Received 18 November 2010; revised 14 June 2011; accepted 6 July 2011; published online 15 September 2011 
Medicine approved the study. We invited all villagers with a minimum age of 12 years to take part. Of 3402 eligible subjects, 2463 (72.4\%) gave informed written consent. We excluded 526 subjects, because their central BP $(n=180)$ or covariables required for analysis $(n=134)$ had not been measured, or because they were taking antihypertensive drugs $(n=212)$. Of 2044 subjects analyzed cross-sectionally, 1224 were re-invited for a repeat examination of their arterial properties, of whom $736(60.1 \%)$ took part. Of these, we excluded 37, because they were taking BP lowering drugs at follow-up. Thus, the number of subjects available for cross-sectional and longitudinal analyses totaled up to 2044 and 699 , respectively.

\section{Measurement of peripheral and central BP}

To ensure a steady state, measurements of peripheral and central BPs were obtained at a local examination center after subjects had rested for at least $5 \mathrm{~min}$ in the supine position. Participants refrained from smoking, heavy exercise and drinking alcohol or caffeinated beverages for $2 \mathrm{~h}$ or longer before the examination.

Trained observers $(n=3)$ did the arterial measurements. During an 8 -s period, they recorded the radial arterial waveform at the dominant arm by applanation tonometry. They used a high-fidelity SPC-301 micromanometer (Millar Instruments, Inc., Houston, TX, USA) interfaced with a laptop computer, running the SphygmoCor software version 6.3.1, and from 2007 onwards, version 7.1 (AtCor Medical Pty. Ltd., West Ryde, New South Wales, Australia). Recordings were discarded when the systolic or diastolic variability of consecutive waveforms exceeded $5 \%$, or when the amplitude of the pulse wave signal was below $80 \mathrm{mV}$. The radial pulse wave was calibrated by the supine brachial BP, which was measured immediately before the tonometric recordings. From the radial signal, the SphygmoCor software calculates the aortic pulse wave by means of a validated ${ }^{12,13}$ generalized transfer function. The software returns the central SBP and the pressure at the first (P1) and second (P2) peak or shoulder of the central and radial waveforms. By repeat examination of 16 subjects at a mean time interval of $1 \mathrm{~h}$, we computed the coefficient of variation as the ratio of the mean difference between the two repeat measurements to the s.d. of the within-subject differences multiplied by $100 .{ }^{14}$ Intra-observer coefficients of variability for central SBP, P1 and P2 were $2.7,1.1$ and $8.9 \%$, respectively.

For the present analysis, peripheral SBP was the average of the three BP readings obtained at the brachial artery for calibration of the radial pressure waves, with the subjects in the supine position. Central SBP was the maximum pressure of the central waveform. We calculated systolic augmentation by subtracting P1 from SBP. Pressure amplification was defined as peripheral minus central SBP. The peripheral (radial) and central augmentation indexes were the ratios of the second to the first peak of the pressure wave expressed in percent. The peripheral (brachial) BP was measured by means of the validated ${ }^{15}$ OMRON 705CP oscillometric sphygmomanometer, fitted with a standard cuff with an inflatable bladder of $22 \times 12 \mathrm{~cm}$ (Omron Inc., Kyoto, Japan). Hypertension was a BP of at least $140 \mathrm{~mm} \mathrm{Hg}$ systolic or $90 \mathrm{~mm} \mathrm{Hg}$ diastolic.

\section{Other measurements}

We administered a standardized questionnaire to obtain information on each subject's medical history, smoking and drinking habits, and use of medications. Venous blood was collected after overnight fasting. We measured total serum cholesterol and blood glucose by automated enzymatic methods. Body mass index was body weight in kilograms divided by height in meters squared. Diabetes mellitus was a fasting blood glucose of at least $7.0 \mathrm{mmoll}^{-1}$ $\left(126 \mathrm{mg} \mathrm{dl}^{-1}\right)$ or the use of anti-diabetic drugs.

\section{Statistical methods}

For database management and statistical analysis, we used SAS software, version 9.1.3 (SAS Institute, Cary, NC, USA). For comparison of means and proportions, we applied the large-sample $Z$-test and the $\chi^{2}$-statistic, respectively. For the assessment of the cross-sectional associations between BP and age, we applied regression analyses. We determined significance of pressure amplification, systolic augmentation pressure and the longitudinal trends in the hemodynamic measurements by dividing the group mean differences or changes in SBP by the s.e. Statistical significance was a $P$-value of 0.05 or less on two-sided tests. We searched for variables associated with the peripheral and central SBPs, using the stepwise linear regression. We set the $P$-values for variables to enter and to stay in the regression models at 0.05 . We tested the null hypothesis of no differences between the regression slopes of hemodynamic measurements on age, using multivariable analysis of variance as implemented in the MTEST statement of the PROC REG procedure of the SAS package. ${ }^{16}$

\section{RESULTS}

\section{Characteristics of participants}

The 2044 participants included 1066 women (52.2\%) and 556 hypertensive patients $(29.0 \%)$, of whom by design of the analysis, none was taking antihypertensive drugs. Age ranged from 12-94 years. Table 1 gives the anthropometric characteristics, risk factors and the peripheral and central hemodynamic measurements by sex. Of 2044 participants, 614 (30.0\%; all men) were current smokers and 907 (44.4\%; 240 women and 667 men) reported regular alcohol intake. In the current smokers, median tobacco use was 20 cigarettes per day (interquartile range, 15-20). In participants reporting alcohol intake, median alcohol intake was $14 \mathrm{~g}$ per day (interquartile range, 6-21) among women and $38 \mathrm{~g}$ per day (18-69) in men. Among 115 participants who reported having cardiovascular diseases at enrollment, 83 had hypertension, 11 had arrhythmia, 7 had ischemic heart disease, 4 had cerebral infarction, 3 had rheumatic heart disease and 7 had various other cardiovascular diseases. Peripheral systolic, diastolic and pulse pressures were significantly higher in men than women $(P \leqslant 0.027)$, but the corresponding central pressures were all similar in both sexes $(P \geqslant 0.76)$. The peripheral and central augmentation indexes were higher in women than men $(P \leqslant 0.001)$.

Of 1066 women and 978 men, 369 (34.6\%) and 330 (33.7\%), respectively, underwent a repeat examination at a median interval of 3.60 years (5th-95th percentile interval, 3.56-3.96 years). Table 1 also provides the baseline characteristics of this subsample by sex. The characteristics of the subsample were similar to those of the whole study group.

\section{Associations of peripheral and central SBP with age}

In cross-sectional analyses of 1066 women and 978 men, the peripheral and central SBPs increased with age ( $P$ for trend $<0.0001$;. Figure 1 , panels a and b). Regression analysis showed that the cross-sectionally assessed age-related increase in SBP was larger $(P<0.001)$ at central than peripheral arteries, both in women (1.07 vs. $0.95 \mathrm{~mm} \mathrm{Hg}$ per year) and in men ( 0.76 vs. $0.59 \mathrm{~mm} \mathrm{Hg}$ per year; Table 2). SBP increased more with age at central than peripheral arteries in women below age 50 (1.21 vs. $1.01 \mathrm{~mm} \mathrm{Hg}$ per year; $P<0.001$ ) and in men below age 60 ( 0.73 vs. $0.48 \mathrm{~mm} \mathrm{Hg}$ per year; $P<0.001$ ), whereas in older women ( 0.64 vs. $0.58 \mathrm{~mm} \mathrm{Hg}$ per year; $P=0.27$ ) and older men $(0.45$ vs. $0.44 \mathrm{~mm}$ $\mathrm{Hg}$ per year; $P=0.79)$, the slopes of central and peripheral SBPs on age were similar. Compared with men, women had steeper $(P<0.001)$ age-related increases in peripheral and central SBPs (Table 2$)$.

The amplification pressure, the difference of peripheral minus central SBP, decreased with age at a rate of $-0.13 \mathrm{~mm} \mathrm{Hg}$ per year in women and $-0.17 \mathrm{~mm} \mathrm{Hg}$ per year in men (Figure 1, panels $\mathrm{c}$ and $\mathrm{d}$ and Table 2). The systolic augmentation pressure, the difference between the maximal and non-augmented systolic pressure, increased linearly with age, but the slope was smaller $(P<0.001)$ for the peripheral than for the central augmentation pressure: $0.086 v s .0 .45 \mathrm{~mm} \mathrm{Hg}$ per year in women and 0.083 vs. $0.39 \mathrm{~mm} \mathrm{Hg}$ per year in men. In both women (12-29 vs. 30-39 years) and men (12-29 vs. 40-49 years), central systolic augmentation occurred at a younger age than peripheral systolic augmentation (30-39 vs. 60-69 years; Figure 1, panels e and f). 
Table 1 Baseline characteristics of participants by study phase and sex

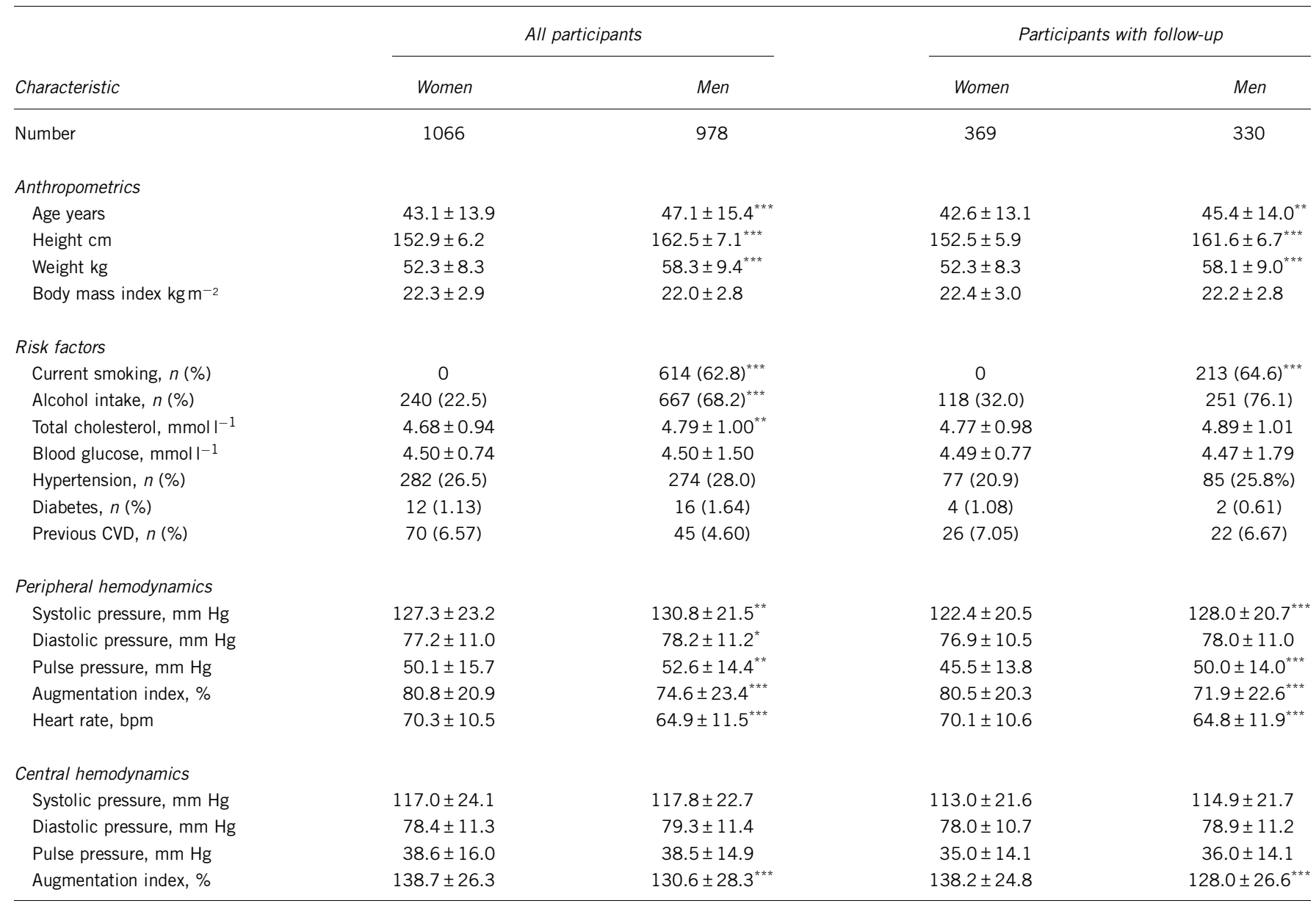

Abbreviation: CVD, cardiovascular disease

Values are mean \pm s.d. or numbers of subjects (\%). Hypertension was a blood pressure of at least $140 \mathrm{~mm} \mathrm{Hg}$ systolic or $90 \mathrm{~mm} \mathrm{Hg} \mathrm{diastolic.}$

Significance of the sex difference ${ }^{*} P<0.05$, ${ }^{* *} P<0.01$ and ${ }^{* * *} P<0.001$.

A subsample of 369 women and 330 men underwent a repeat assessment of peripheral and central SBP (Figure 2 and Table 2). The slopes on age of the peripheral and central SBPs, amplification pressure, and peripheral and central systolic augmentation pressures were similar at baseline and follow-up $(P \geqslant 0.09)$. In the longitudinal analyses, all changes in SBP from baseline to follow-up were significant $(P<0.001)$. Considering the whole study population, the annual increases in the peripheral and central SBPs were larger in women than men $(P<0.001$, Table 3$)$. However, compared with peripheral SBP, central SBP increased less both in women $(2.12$ vs. $2.35 \mathrm{~mm} \mathrm{Hg}$, $P=0.003)$ and in men $(1.16$ vs. $1.37 \mathrm{~mm} \mathrm{Hg}, P=0.025)$. On a relative scale, the percentage increases in peripheral and central SBPs from baseline to follow-up were similar in women $(2.14 \mathrm{vs} .2 .16 \%$ per year; $P=0.76$ ), as well as in men ( 1.33 vs. $1.34 \%$ per year; $P=0.96$; Table 3 ). In sensitivity analyses stratified by quartiles of the age distribution (Table 3), the increase in peripheral SBP was larger than that in central SBP $(P \leqslant 0.02)$ above median age in women and above the 75 th percentile of age in men, whereas in all other sex-age subgroups, the increases of peripheral and central SBPs were similar $(P \geqslant 0.08)$.

\section{Multivariable analyses}

Table 4 is based on the cross-sectional analysis of the whole study sample at baseline and summarizes the results of stepwise regression analysis with peripheral and central SBPs as the dependent variables. Age contributed most of the explained variance. Adding a quadratic to the linear term of age (Table 4) did not significantly improve the explained variance, except for central SBP in women (partial $r^{2}=0.003$; $P=0.02$ ). The multivariable-adjusted slopes of BP on age were almost similar to those in single regression analysis (Table 2 ). The slope of SBP on age was significantly steeper $(P<0.001)$ for central than peripheral SBP, and in women compared with men.

In both women and men, the peripheral and central SBPs increased with body mass index and central SBP was inversely associated with heart rate. In men, peripheral SBP was on average $2.8 \mathrm{~mm} \mathrm{Hg}$ lower in smokers than non-smokers, whereas drinking alcohol was associated with increases in central and peripheral SBPs by approximately $5 \mathrm{~mm} \mathrm{Hg}$.

\section{DISCUSSION}

The present report focused on the impact of age on central and peripheral SBP and systolic augmentation in randomly recruited Chinese. The main finding in the cross-sectional analysis was that in the young and middle-aged subjects, central SBP increased more with age than peripheral SBP, so that the amplification of systolic pressure from the central to the peripheral arteries substantially decreased with advancing age. However, in older subjects, peripheral and central SBP 
a

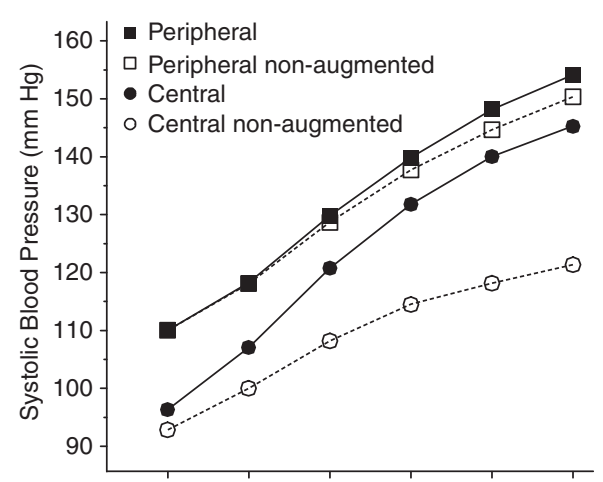

\section{C}

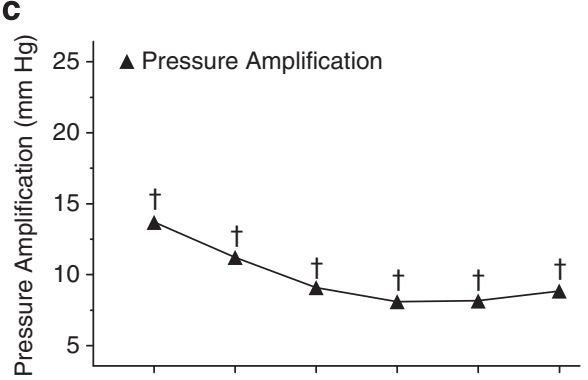

e

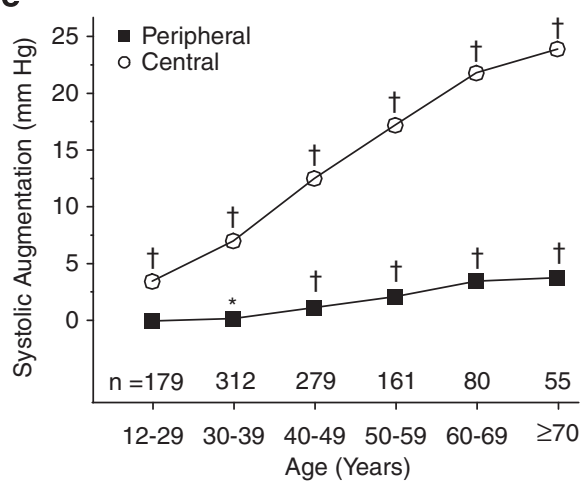

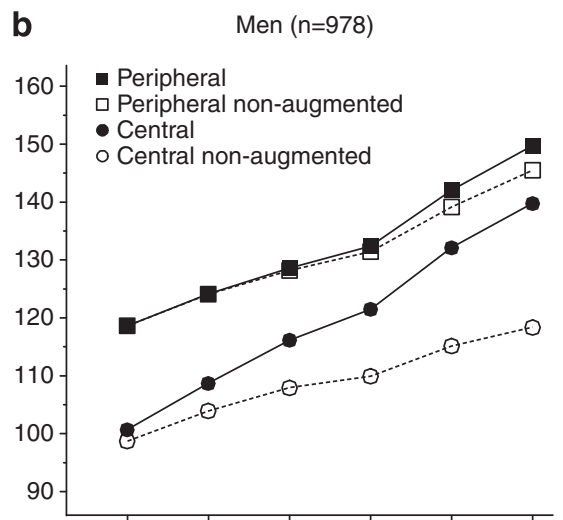

d

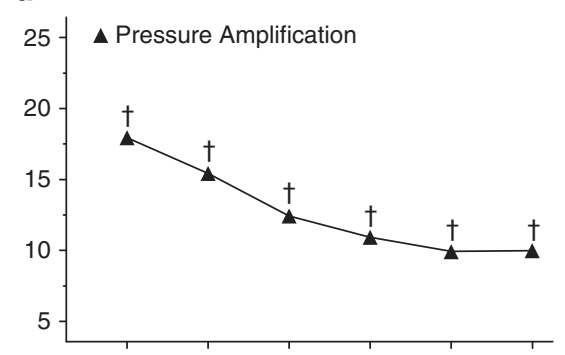

f

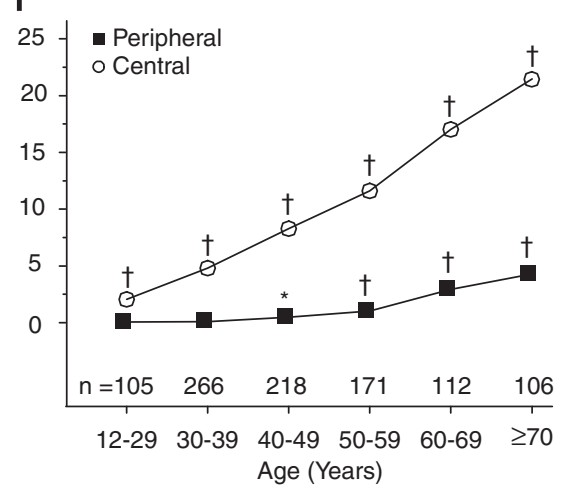

Figure 1 Association with age of peripheral and central SBPs (a, b), peripheral and central non-augmented SBPs (a, b), pressure amplification (c, d), and peripheral and central systolic augmentation $(\mathbf{e}, \mathbf{f})$ in women $(\mathbf{a}, \mathbf{c}, \mathbf{e})$ and men $(\mathbf{b}, \mathbf{d}, \mathbf{f})$. Peripheral SBP was the average of three BP readings at the brachial artery. Central SBP was the maximal pressure of the central waveform. Systolic augmentation was obtained by subtracting the first systolic peak from SBP. Pressure amplification is peripheral minus central SBP. Plotted values are means for each age group. Numbers indicate the subjects contributing to the group means. All $P$-values for trend with age were statistically significant $(P<0.0001)$. Significance of the difference with zero: ${ }^{*} P<0.05$ and $\dagger P<0.001$.

increased in parallel with age with no further reduction of the pressure amplification. The gradient in SBP between the central and peripheral arteries narrowed with advancing age, because systolic augmentation appeared earlier and at a much faster rate in the central than in the peripheral arterial system.

Stiffening of the large arteries underlies the age-related increase in BP. ${ }^{17}$ The loss of arterial elasticity over a person's life time is partly due to cyclic stress on the arterial wall with each heart beat. ${ }^{18}$ Over time, this causes fracture of elastin fibers, so that stress is transferred to the more rigid collagenous components of the arterial wall. At a young age, the aorta and proximal arteries dilate by approximately $10 \%$ with each heart beat, whereas the more distal muscular arteries dilate by only $2-3 \%$ with each heart beat. ${ }^{19}$ Atherosclerosis and inflammation thicken the arterial wall and contribute to arterial stiffening over and beyond the mechanical stress. To differentiate natural degeneration of the arterial wall from aging from disease, Avolio et al. ${ }^{6}$ highlighted the interest of studies of arterial properties in population studies with low cholesterol and low prevalence of atherosclerosis, such as Chinese.

In 1985, Avolio et al. ${ }^{5}$ contrasted the Chinese living in areas with low and high prevalence of hypertension, Guangzhou (4.9\%) and Beijing (15.6\%), respectively. In Guangzhou subjects, pulse wave velocity was consistently lower in the aorta, arm and leg, and increased to a lesser degree with age, compared with Beijing subjects. ${ }^{5}$ The contemporary cholesterol levels were $4.34 \mathrm{mmoll}^{-1}$ in Guangzhou subjects and $4.49 \mathrm{mmoll}^{-1}$ in Beijing subjects. ${ }^{5}$ In our Chinese population, cholesterol levels were of similar magnitude $\left(4.73 \mathrm{mmoll}^{-1}\right)$, but the prevalence of hypertension was much higher (27.2\%). Our current observations strengthen Avolio's hypothesis 5,6 
Table 2 Cross-sectional association of blood pressure with age in women and men

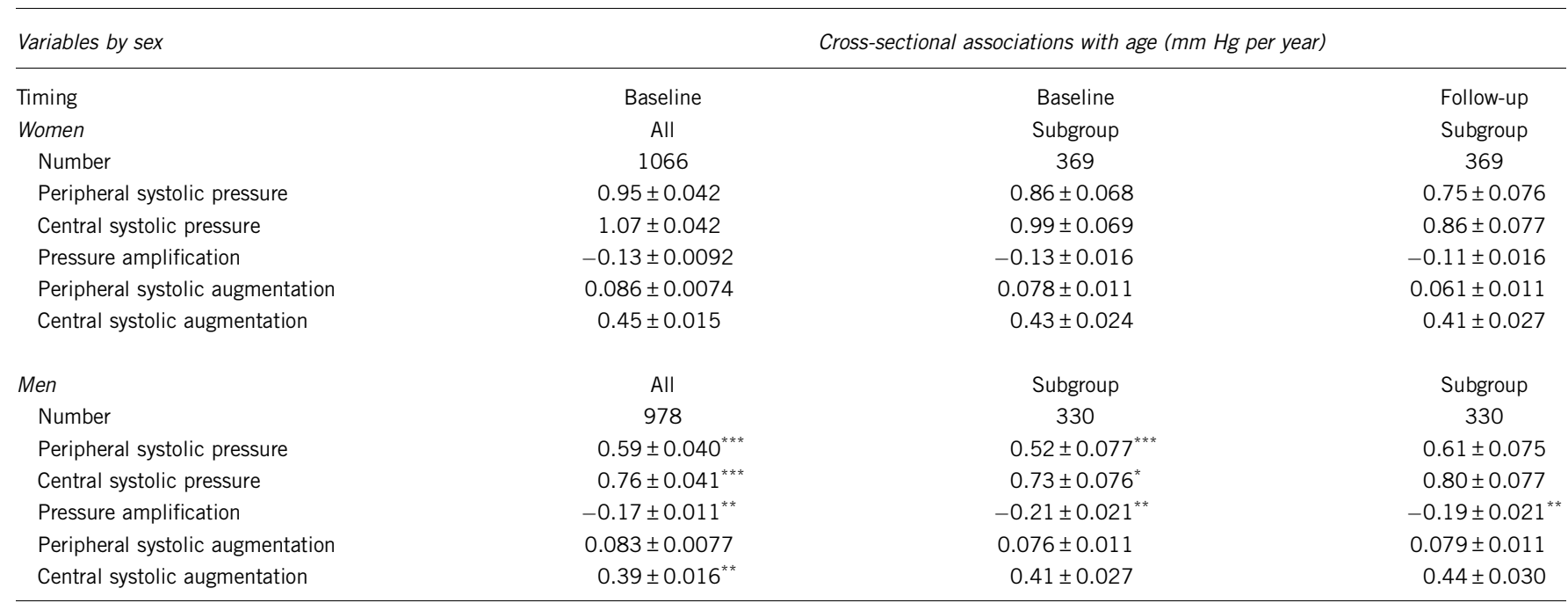

Single regression co-efficients ( $\beta \pm$ s.e.) describe the cross-sectional associations of blood pressure with age. Subgroup refers to the subjects with repeat hemodynamic measurements at a median interval of 3.6 years. Peripheral systolic blood pressure was the average of three blood pressure readings at the brachial artery. Central systolic blood pressure was the maximal pressure of the interval of 3.6 years. Peripheral systolic blood pressure was the average of three blood pressure readings at the brachial artery. Central systolic blood pressure was the maximal pressure of the
central waveform. Systolic augmentation was obtained by subtracting the first systolic peak from systolic blood pressure. Pressure amplification is peripheral minus central systolic blood pressure. All slopes on age were statistically significant $(P<0.001)$. In subjects with repeat examinations, slopes on age did not differ between baseline and follow-up $(P \geqslant 0.09)$. Significance of the sex difference: ${ }^{*} P<0.05,{ }^{* *} P<0.01$ and ${ }^{* * *} P<0.001$.
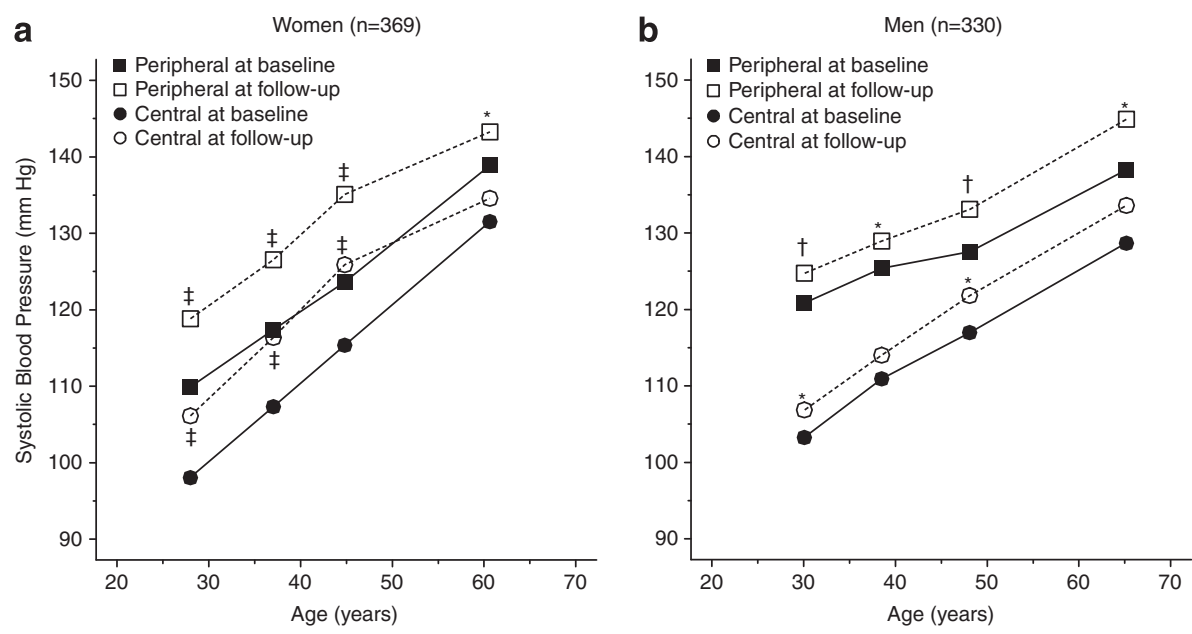

Figure 2 Peripheral and central SBPs at baseline and follow-up by quartiles of the age distribution in 369 women (a) and 330 men (b). All $P$-values for trend with age were statistically significant $(P<0.0001)$. Significance of the difference between baseline and follow-up: ${ }^{*} P<0.05,{ }^{\dagger} P<0.01$ and $\ddagger P<0.001$.

that slowly progressing degeneration of the arterial wall through cyclic stress is the main cause of the age-related increase in SBP. Indeed, in the young and middle-aged subjects, when atherosclerosis is rare among rural Chinese, central SBP increased more than peripheral SBP, whereas in older subjects, central and peripheral SBP increased in parallel with advancing age. From the literature and our observations, it is becoming clear that aging differentially affects the arterial system across the age range and the various sections of the arterial tree. Fatigue of elastin fibers in the proximal elastic arteries has already started in the young and middle-aged subjects, in particular, in the presence of a high SBP. Breakdown of the elastic lamellae in elastic arteries explains why in the young and middle-aged subjects central SBP increases at a faster rate than peripheral SBP. At older age, when more of the SBP load is less dampened at elastic arteries and is transmitted into the more distal muscular arteries, central and peripheral SBP continue to rise, but in a parallel fashion.

In the young and middle-aged subjects, the age-related increase in SBP ran a steeper course in women than men. At all ages, women have a higher heart rate than men. ${ }^{20}$ The smaller height of women may be a cardiovascular risk factor, because of the early return of reflected waves to the central aorta in systole rather than diastole. The shorter stature of women also implies reduced length of the arterial tree, a factor believed to be responsible for the faster heart rate, a shorter diastolic period, a shorter diastolic time constant, and at the same peripheral resistance, lower arterial compliance. ${ }^{20}$ In older women, menopause might contribute to the continuing rise in SBP, although it is difficult to differentiate the effects of aging from those of estrogen deprivation. ${ }^{21,22}$ 
Table 3 Longitudinal change in blood pressure with aging in women and men

\begin{tabular}{|c|c|c|c|c|c|}
\hline \multirow[b]{2}{*}{ Variables by sex } & \multicolumn{4}{|c|}{ Age quartile } & \multirow[b]{2}{*}{ All } \\
\hline & Q1 & Q2 & Q3 & Q4 & \\
\hline Baseline age range, years & $\leqslant 33.1$ & $33.2-40.1$ & $40.2-50.1$ & $50.2-80.0$ & $12.0-80.0$ \\
\hline Number & 93 & 92 & 92 & 92 & 369 \\
\hline \multicolumn{6}{|l|}{ Peripheral systolic pressure } \\
\hline \multicolumn{6}{|l|}{ Central systolic pressure } \\
\hline Change, mm Hg per year & $2.23 \pm 3.46$ & $2.52 \pm 4.10$ & $2.91 \pm 4.44^{*}$ & $0.81 \pm 5.84^{* *}$ & $2.12 \pm 4.59^{* *}$ \\
\hline Relative change, \% per year & $2.42 \pm 3.45$ & $2.47 \pm 3.76$ & $2.66 \pm 3.87$ & $1.09 \pm 4.46$ & $2.16 \pm 3.93$ \\
\hline \multicolumn{6}{|l|}{ Men } \\
\hline Baseline age range, years & $\leqslant 34.6$ & $34.7-41.8$ & $41.9-53.5$ & $53.6-82.5$ & $14.2-82.5$ \\
\hline \multicolumn{6}{|l|}{ Central systolic pressure } \\
\hline Change, $\mathrm{mm} \mathrm{Hg}$ per year & $0.99 \pm 3.83$ & $0.88 \pm 4.16$ & $1.38 \pm 5.06$ & $1.38 \pm 7.00^{*}$ & $1.16 \pm 5.14^{*}$ \\
\hline Relative change, \% per year & $1.21 \pm 3.67$ & $1.01 \pm 3.67$ & $1.49 \pm 4.43$ & $1.63 \pm 5.81$ & $1.34 \pm 4.46$ \\
\hline
\end{tabular}

All changes from baseline to follow-up were significant $(P<0.05)$. Change was the average blood pressure change per year (follow-up minus baseline). Relative change was the percentage change per year relative to the corresponding level at baseline.

Significance of the difference between central and peripheral pressure changes: ${ }^{*} P<0.05 ;{ }^{* *} P<0.01$.

Table 4 Correlates of peripheral and central SBPs in women and men

\begin{tabular}{|c|c|c|c|c|c|c|}
\hline & \multicolumn{3}{|c|}{ Women $(n=1066)$} & \multicolumn{3}{|c|}{ Men $(n=978)$} \\
\hline & Peripheral & Central & P-values & Peripheral & Central & P-values \\
\hline$r^{2}$ & 0.34 & 0.40 & & 0.20 & 0.29 & \\
\hline Intercept & 65.6 & 62.7 & & 84.5 & 78.9 & \\
\hline \multicolumn{7}{|l|}{ Age, years } \\
\hline$\beta \pm$ s.e. & $0.94 \pm 0.042^{* * *}$ & $1.04 \pm 0.043^{* * *}$ & $<0.001$ & $0.61 \pm 0.040^{* * *}$ & $0.75 \pm 0.040^{* * *}$ & $<0.001$ \\
\hline Partial $r^{2}$ & 0.32 & 0.38 & & 0.18 & 0.26 & \\
\hline \multicolumn{7}{|c|}{ Body mass index, $\mathrm{kg} \mathrm{m}^{-2}$} \\
\hline$\beta \pm$ s.e. & $0.94 \pm 0.20^{* * *}$ & $0.88 \pm 0.20^{* * *}$ & 0.10 & $0.72 \pm 0.23^{* *}$ & $0.51 \pm 0.23^{*}$ & 0.0056 \\
\hline Partial $r^{2}$ & 0.014 & 0.011 & & 0.010 & 0.0056 & \\
\hline \multicolumn{7}{|c|}{ Heart rate, bpm } \\
\hline$\beta \pm$ s.e. & NS & $-0.15 \pm 0.056^{\dagger}$ & - & NS & $-0.21 \pm 0.054^{* * *}$ & - \\
\hline Partial $r^{2}$ & & 0.0044 & & NS & 0.011 & \\
\hline \multicolumn{7}{|c|}{ Smoking $(0,1)$} \\
\hline$\beta \pm$ s.e. & - & - & - & $-2.80 \pm 1.31^{*}$ & NS & - \\
\hline Partial $r^{2}$ & & & & 0.0037 & NS & \\
\hline \multicolumn{7}{|c|}{ Drinking alcohol $(0,1)$} \\
\hline$\beta \pm$ s.e. & NS & NS & - & $4.94 \pm 1.34^{* * *}$ & $5.03 \pm 1.32^{* * *}$ & 0.13 \\
\hline Partial $r^{2}$ & & & & 0.0002 & 0.012 & \\
\hline
\end{tabular}

Abbreviation: NS, not significant

Abbreviation: NS, not significant
Values are partial regression coefficients $\left(\beta \pm\right.$ s.e.) or partial coefficients of determination $\left(r^{2}\right)$. Significance of the partial regression coefficient: NS $P \geqslant 0.05 ;{ }^{*} P<0.05$; ${ }^{* *} P<0.01$ and ${ }^{* * *} P<0.001$. The covariables considered in the stepwise regression procedure were: age, body mass index, current smoking and drinking, and heart rate. $P$-values are for the comparison of the partial regression coefficients describing the associations with peripheral vs. central blood pressure. An ellipsis indicates not applicable. 
Our cross-sectional observations are in line with previously published population studies in Caucasians. ${ }^{7,11}$ McEniery et al. ${ }^{7}$ measured the peripheral and central SBP and augmentation pressure in 4001 healthy, normotensive individuals, aged 18-90 years. As we did, McEniery's team also used the SphygmoCor device. Subjects with hypertension ( $\mathrm{BP} \geqslant 140 / 90 \mathrm{~mm} \mathrm{Hg}$ ), diabetes mellitus, serum cholesterol $\geqslant 6.5 \mathrm{mmoll}^{-1}$, renal disease (defined as a clinical history, creatinine $\geqslant 150 \mu \mathrm{moll}^{-1}$ or active urinary sediment), or cardiovascular disease (defined as a clinical history or evidence on examination) were excluded from the analysis, as were subjects receiving any medication. In both women and men, central systolic pressure increased more with age than did peripheral SBP $(P<0.001)$, and, as in our study, this trend was evident in young and middle-aged subjects, and the increase in central systolic pressure was more prominent in women than in men $(P=0.01) .^{7}$ In the Framingham Third Generation and Offspring study, ${ }^{23}$ peripheral and central SBP increased little from below 30 up to 50 years of age, and thereafter, ran a similar course, both on average increasing from $120 \mathrm{~mm} \mathrm{Hg}$ to approximately $150 \mathrm{~mm} \mathrm{Hg}$.

In our study, the longitudinal estimates of the age-related increases in peripheral and central SBP were consistently higher than on crosssectional assessment. In an early Framingham report, ${ }^{24}$ Kannel and Gordon $^{24}$ also noticed that the age-related increase in SBP was steeper on cross-sectional than longitudinal assessment in women, whereas the opposite was the case in men. The reasons for the difference in BP trends obtained cross-sectionally and longitudinally in the same Framingham cohort were not clear.

Our study should be interpreted keeping in mind some potential limitations. First, the sample size in our longitudinal study was small and the longitudinal estimates of SBP were based on no more than two measurements at a median interval of 3.6 years. In the aforementioned Framingham report, ${ }^{24}$ the longitudinal data were based on eight biennial examinations. Second, in our longitudinal analysis, at variance with the cross-sectional analysis, the increase in central SBP was not larger than the increase in peripheral SBP. When central BP waveforms are calibrated by brachial cuff pressures, the SphygmoCor approach might underestimate central SBP, in particular, at high levels of SBP. ${ }^{25-26}$ However, pressure amplification, when expressed as the ratio of peripheral divided by central pulse pressure, should be independent of possible calibration errors. ${ }^{27}$ As shown in the Supplementary Table available online, pressure amplification ratio (peripheral/central pulse pressure) consistently decreased with age in both cross-sectional and longitudinal analyses.

The effects of aging on arterial function have often been underestimated, because of the sole reliance on the brachial cuff systolic pressure. In our current study, we measured both peripheral and central SBP. In the young and middle-aged subjects, central SBP increased more with age than peripheral SBP. Our current findings support the viewpoint that early in life, the cyclic systolic load on the arteries has already caused degenerative changes in the arterial wall and, as the years go by, causes arterial stiffening. This process represents a vicious circle, in which increasing SBP is, at the same time, the cause and the consequence of a self-sustaining process that leads to major cardiovascular complications. Breaking the vicious circle is the key to slowing the age-related rise in SBP and preventing the associated cardiovascular complications. Thus, our present findings highlight the clinical importance of timely diagnosis and treatment for hypertension, in particular systolic hypertension. From the research point of view, our longitudinal observations need to be replicated in studies with multiple re-assessment of arterial properties over time.

\section{CONFLICT OF INTEREST}

The authors declare no conflict of interest.

\section{ACKNOWLEDGEMENTS}

The study was financially supported by the National Natural Science Foundation of China (grants 30871360 and 30871081), the Ministry of Science and Technology (grant 2006BAI01A03 and a 2010 China-EU collaboration grant [1012]), and the Ministry of Education (New Century Excellent Talents in Universities, NCET-09-0544), Beijing, China. Support was also provided by the Shanghai Commission of Science and Technology (grants 05ZR14100 and 07JC14047 and the 'Rising Star' programs 06QA14043 and 11QH1402000), the Shanghai Commission of Education (grant 07ZZ32 and the 'Dawn' project 08SG20), the Shanghai Shenkang Hospital Development Center (grant SHDC12007318), the Shanghai Jiaotong University School of Medicine (Distinguished Young Investigator grant to Yan $\mathrm{Li}$ ), and the European Union (grants LSHM-CT-2006-037093 and HEALTH-F4-2007-201550). The authors gratefully acknowledge the voluntary participation of all study subjects, the support of the local public health authorities of JingNing County (ZheJiang Province, China) and the technical assistance of Shou-Yu Mao, Wei-Zhong Zhang, Gu-Liang Wang and Jie Wang (The Shanghai Institute of Hypertension, Shanghai, China).

1 O'Rourke MF, Kelly PJ. Wave reflections in the systemic circulation and its implications in ventricular function. J Hypertens 1993; 11: 327-337.

2 O'Rourke M. Arterial haemodynamics and ventricular-vascular interaction in hypertension. Blood Press 1994; 3: 33-37.

3 Murgo JP, Westerhof N, Giolma JP, Altobelli SA. Aortic input impedance in normal man relationship to pressure wave forms. Circulation 1980; 62: 105-116.

4 Wilkinson IB, MacCallum H, Flint L, Cockroft JR, Newby DE, Webb DJ. The influence of heart rate on augmentation index and central arterial pressure in humans. J Physio 2000; 525: 263-270.

5 Avolio AP, Deng FA, Li WQ, Luo YF, Huang ZD, Xing LF, O'Rourke MF. Effects of aging on arterial distensibility in populations with high and low prevalence of hypertension: comparison between urban and rural communities in China. Circulation 1985; 71: 202-210.

6 Avolio AP, Chen SG, Wang RP, Zhang CL, Li MF, O'Rourke MF. Effects of aging on changing arterial compliance and left ventricular load in a northern Chinese urban community. Circulation 1983; 68: 50-58.

7 McEniery CM, Yasmin, Hall IR, Qasem A, Wilkinson IB, Cockroft JR, ACCT Investigators. Normal vascular aging : differential effects on wave reflection and aortic pulse wave velocity. The Anglo-Cardiff Collaborative Trial (ACCT). J Am Coll Cardiol 2005; 46: 1753-1760.

8 Mitchell GF, Parise H, Benjamin EJ, Larson MG, Keyes MJ, Vita JA, Vasan RS, Levy D. Changes in arterial stiffness and wave reflection with advancing age in healthy men and women. The Framingham Heart Study. Hypertension 2004; 43: 1239-1245

9 Li Y, Staessen JA, Li LH, Huang QF, Lu L, Wang JG. Reference values for the arterial pulse wave in Chinese. Am J Hypertens 2008; 21: 668-673.

10 Wilkinson IB, Franklin SS, Hall IR, Tyrrell S, Cockcroft JR. Pressure amplification explains why pulse pressure is unrelated to risk in young subjects. Hypertension 2001; 38: 1461-1466.

11 McEniery CM, Yasmin, McDonnell B, Munnery M, Wallace SM, Rowe CV, Cockcroft JR, Wilkinson IB, Anglo-Cardiff Collaborative Trial Investigators. Central pressure : variability and impact of cardiovascular risk factors. The Anglo-Cardiff Collaborative Trial II. Hypertension 2008; 51: 1-7.

12 Karamanoglu M, O'Rourke MF, Avolio AP, Kelly PJ. An analysis of the relationship between central aortic and peripheral upper limb pressure waves in man. Eur Heart $J$ 1993; 14: 160-167.

13 Pauca AL, O'Rourke M, Kon ND. Prospective evaluation of a method for estimating ascending aortic pressure from the radial artery pressure waveform. Hypertension 2001; 38: 932-937.

14 Bland JM, Altman DG. Statistical methods for assessing agreement between two methods of clinical measurement. Lancet 1986; 1: 307-310.

15 O'Brien E, Mee F, Atkins N, Thomas M. Evaluation of three devices for self-measurement of blood pressure according to the revised British Hypertension Society Protocol : the Omron HEM-705 CP, Philips HP5332 and Nissei DS-175. Blood Press Monit 1996; 1: 55-62.

16 Staessen J, O'Brien E, Atkins N, Bulpitt CJ, Cox J, Fagard R, O'Malley $K$, Thijs L, Amery $A$. The increase in blood pressure with age and body mass index is overestimated by conventional sphygmomanometry. Am J Epidemiol 1992; 136: 450-459.

17 Staessen J, Amery A, Fagard R. Editorial review. Isolated systolic hypertension in the elderly. J Hypertens 1990; 8: 393-405. 
18 O'Rourke MF, Hashimoto J. Mechanical factors in arterial aging. A clinical perspective. J Am Coll Cardiol 2007; 50: 1-13.

19 Boutouyrie PH, Laurent S, Benetos A, Girerd XJ, Hoeks APG, Safar ME. Opposing effects of ageing on distal and proximal large arteries in hypertensives. $J$ Hypertens 1992; 10: S87-S91.

20 Smulyan H, Asmar RG, Rudnicki A, London GM, Safar ME. Comparative effects of aging in men and women on the properties of the arterial tree. J Am Coll Cardiol 2001; 37: $1374-1380$.

21 Staessen JA, Bieniaszewski L, Brosens I, Fagard R. The epidemiology of menopause and its association with cardiovascular disease. In Messerli FH, Aepfelbacher FC (eds), Hypertension in Postmenopausal Women. Marcel Dekkers Inc: New York, USA, 1996, pp 43-78.

22 Casiglia E, Tikhonoff V, Caffi S, Bascelli A, Schiavon L, Guidotti F, Saugo M, Giacomazzo M, Martini B, Mazza A, D'Este C, Pessina AC. Menopause does not affect blood pressure and risk profile, and menopausal women do not become similar to men. J Hypertens 2010; 26: 1983-1992.
23 Mitchell GF, Wang N, Palmisano JN, Larson MG, Hamburg NM, Vita JA, Levy D, Benjamin EJ, Vasan RS. Hemodynamic correlates of blood pressure across the adult age spectrum : noninvasive evalauation in the Framingham Heart Study. Circulation 2010; 122: 1379-1386.

24 Kannel WB, Gordon T. Evaluation of cardiovascular risk in the elderly : The Framingham Study. Bull N Y Acad Med 1978; 54: 573-589.

25 Zuo JL, Li Y, Yan ZJ, Zhang RY, Shen WF, Zhu DL, Gao PJ, Chu SL. Validation of the central blood pressure estimation by the SphygmoCor system in Chinese. Blood Press Monit 2010; 15: 268-274.

26 Papaioannou TG, Lekakis JP, Karatzis EN, Papamichael CM, Stamatelopoulos KS, Protogerou AD, Mavrikakis M, Stefanadis C. Transmission of calibration errors (input) by generalized transfer functions to the aortic pressures (output) at different hemodynamic states. Int J Cardiol 2006; 110: 46-52.

27 Avolio AP, Van Bortel LM, Boutouyrie P, Cockcroft JR, McEniery CM, Protogerou AD, Roman MJ, Safar ME, Segers P, Smulyan H. Role of pulse pressure amplification in arterial hypertension: experts' opinion and review of the data. Hypertension 2009; 54: 375-383.

Supplementary Information accompanies the paper on Hypertension Research website (http://www.nature.com/hr) 